\title{
Preliminary Measures to Cultivate Students' Researching Study Ability in Comprehensive Experiment
}

\author{
Shu-Qin WANG ${ }^{\mathrm{a},{ }^{*}}$ and Dan LI \\ Department of Environmental Science and Engineering, North China Electric Power University, \\ Baoding 071003, China \\ awsqhg@163.com \\ ${ }^{*}$ Corresponding author
}

\begin{abstract}
Keywords: Research-based teaching, Comprehensiveness experiment, Experimental reform, Researching study ability.

Abstract. The concept and features of research-based teaching were carried over to comprehensive experimental reform process, which emphasize the student's initiative and the design of the experiment process. The research and comprehensiveness of the experimental process have been highlighted, and students' consciousness of active and independent experiments can be enhanced, the thinking habits of solving engineering problems with relevant knowledge can be established; Through the contrast test, repeated experiments and exploration, the students' innovative consciousness and scientific working attitude were cultivated. The whole process' training, from information finding, scheme selection to the experimental operation, made students develop the habit of actively exploring the problem, find the solution in a comprehensive way, and reasonably divide the division of labor, jointly overcome the difficulties, and form persistent team cooperation consciousness.
\end{abstract}

\section{Introduction}

Research-based teaching refers to the process of teaching, students under the guidance of teachers, scientific way to identify problems, analyze problems and solve the problem of the whole process. It breaks the monopoly of scientific research by teachers in the traditional teaching mode. It's open to students, more attention payed to let students independent research, to promote the development of students' innovative ability[1].

Compared with the traditional confirmatory experimental teaching, the difference of the research experimental teaching is that the research questions are not fixed and the experimental results are unknown. In the whole process of experiment, students required to use their own brains to optimize their experimental design, which is conducive to cultivating students' autonomous learning ability and scientific research thinking ability, thereby enhancing their innovative ability. It is more important to improve the students' comprehensive ability greatly, to achieve the "research in the experiment, experiment in the research", to improve students' initiative and enthusiasm for the experiment, to further promote students' understanding and mastery of professional knowledge in experiment[2].

The comprehensive experiment course of Environmental Engineering separately was setted as a link of comprehensive practice teaching. Through years of teaching reform, the research teaching methods, experimental design optimization and monitoring of the implementation process have been effective combined. Thus, the systematicness of students' knowledge of professional knowledge have been enhanced, students' hands-on ability and scientific research accomplishment have been trained. It has played a decisive role in cultivating students' practical ability, deepening the understanding and application of theoretical knowledge and developing innovation. Mainly reflected in the following two aspects. 


\section{Main Features of Research-oriented Teaching in Environmental Engineering Comprehensive Experimental Course}

\section{Student Initiative and the Design of the Experimental Process Emphasizes}

In traditional experimental teaching, teachers are often keen to teach students the purpose of the experiment, experimental principles, experimental procedures, experimental devices, data processing methods and experimental considerations, etc. Students, like "robot", copy the same experimental procedure and order of teachers, step by step operation[3]. Due to the lack of proper thinking about the principle of the experiment and the operation of the instrument, the professional knowledge involved can not be transferred and applied flexibly in the experimental environment. Research teaching requires students to actively study and explore in the environment created by teachers. Students are the subject of understanding, finding and solving problems[4]. In this course, teachers should be good at arousing students' hunger after knowledge, let students actively explore the principles, steps, devices and data processing methods of experiment, so that students actively participate in the whole process of experiment[5].

According to the existing experimental conditions, we determine several different comprehensive experimental topics and give the task requirements respectively. Students are grouped into small groups, each with 5-6 person, select the responsible student as leader, the leader divides the team members according to the task of instructors. Reasonable grouping is a prerequisite for the smooth development, taking into account the students' ability differences, gender differences, personality differences, reasonable collocation, uniform distribution, so that students will achieve better cooperation between the members. Before the experiment begins, students need to check the databases of "CNKI", "Wanfang", "Ei compendex", "Elsevier", "Springer", carefully review research reports or experimental papers similar to this group's experimental tasks, and design the experimental scenarios for this group in advance, based on the existing replication experiments[6]. The types and quantities of laboratory chemicals and instruments, experimental methods and experimental steps used by each group are selected alone. The student may choose the different processing object to carry on the monitoring management, may also choose the same processing object to carry on the different processing units or the different processing methods for effective contrast, requests each group to communicate adequately, avoids duplication and plagiarism. After determined, the experimental scheme was corrected by the teacher.

\section{Highlight the Research and Comprehensiveness of The Experimental Process}

The research experiment is a design, application and development experiment with exploratory properties, also known as exploratory (inquiry) experiment. In traditional experimental teaching mode, the teachers not only listed the experimental purposes and steps, but also clarified the quantity of the experimental equipment and chemicals, the purity of the reagent, the experimental method and even the assembly of the instruments[7]. In the research experimental teaching mode, emphasis on the interaction between teachers and students; teachers help students in accordance with experimental purposes, let students use their own professional knowledge and the new knowledge retrieved on the Internet, fully exchange and cooperation with members of the same group, so that students determine the type and number of experimental chemicals and equipments, experimental methods, procedures and sequences, to develop students' comprehensive abilities in the study[8].

In this course, the roles of students and teachers have changed, students are the center of teaching activities and the subject of experiment, teachers are the organizers and coordinators, to give full play to students' subjective initiative, to exercise their ability to complete the experiment independently[9].

In the course of the experiment, the students asked to not only measure the multiple indexes, but also for multiple processing units or a variety of treatment methods, the effect of contrast, required to sample themselves, determine the number of laboratory equipment and chemicals, the purity of reagents, assemble the necessary equipment, prepare their own solution required themselves, after completing all the preparatory work, carry out the test separately. The team leader is responsible for organizing and coordinating, arranging the experiment sequence, communicating problems with the instructor in time, and actively accessing the relevant data to revise the experiment program. 


\section{Improvement Measures for Research Learning Ability of Environmental Engineering Comprehensive Experimental Course}

\section{Analyze the Causes of Engineering Problems by Experiment and Propose Solutions to Improve Engineering Practice Ability}

Putting forward questions is the starting point of learning, solving problems is the driving force of learning and the main thread of learning process. For this, we ask students with problems, to arrange time well, grasp every link and carry out experiments independently.

For example, in the experimental project of "analysis of the causes of sludge bulking in the $\mathrm{A}_{2} / \mathrm{O}$ process and the countermeasures for improvement", students through the visit to on-campus Sewage treatment station to feel the phenomenon of sludge bulking, retrieve water samples and activated sludge, develop experimental program, analyse the total number of microorganisms of samples by the knowledge of the previous courses such as: Environmental Monitoring, Microbiology of Environmental Engineering, Water Pollution Control Engineering, and comparative analysis with the sludge of the sewage treatment plant without sludge bulking (Fig.1). It was found that the sludge activity of the on-campus Sewage treatment station was poor, the microbial species were few and no filamentous bacteria appeared, so the judgment is the sludge bulking caused by non-filamentous bacteria. And for the sludge index SVI test found that its value is greater than 130, sludge settling performance is poor, also analyzed the ratio of carbon, nitrogen, phosphorus in aerobic pool, found that the phosphorus content is higher significantly, the $\mathrm{pH}$ value is low, and the dissolved oxygen content is less than $2 \mathrm{mg} / \mathrm{L}$, which are the cause of non filamentous sludge bulking. According to the professional knowledge, put forward adjustment measures, reduce emissions of phosphorus-containing wastewater or add organic matter to improve the nutrition ratio and $\mathrm{pH}$ of water; Reduce the amount of aeration, under the condition of low water temperature in winter. The operation effect of reducing aeration is as follows (Fig.2), although there are some bubbles, but no overflow aeration pool, the pool reaction uniformity, sludge expansion has been significantly improved.

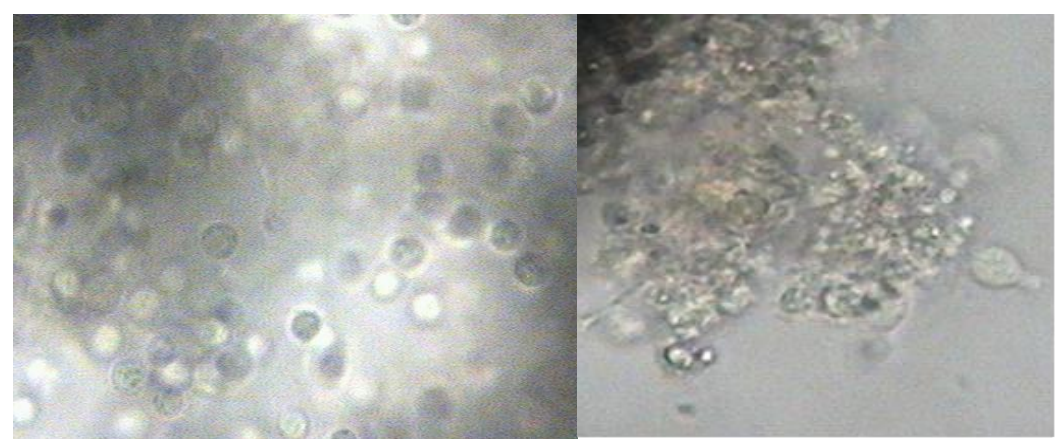

Fig.1 Sludge activity of off-campus sewage treatment plant (left) and on-campus Sewage treatment station (right)

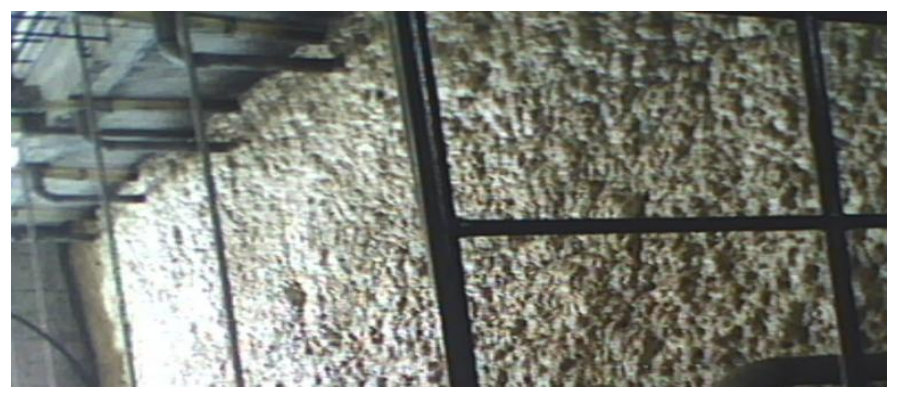

Fig.2 Operation effect after reducing aeration

Through a two-week comprehensive experiment, the students conduct multi-index testing, analysis and governance research on the actual problems that occurred in on-campus Sewage treatment station, strengthen understanding of the $\mathrm{A}_{2} / \mathrm{O}$ process and learned to use the knowledge of the professional courses to solve practical problems, exercise and improve the engineering practice ability. 


\section{Improve the Ability to Analyze and Solve Problems by Comparing Tests}

In the process of actual operation, there are various problems and difficulties are unavoidable. Teachers should instruct students to face problems and difficulties correctly, cultivate students' ability to solve problems, and also help them develop their sound personalities. For example, in experiment "coagulation, adsorption treatment of domestic sewage", some students found that the same coagulant had different effects on turbidity and chromaticity after treating different sewage. The effects of ferric salts and aluminum salts in the treatment of the same wastewater are also different. After the coagulation by iron salt, the floc is large, but the residual chromaticity is high; But the alum floc is small when the aluminum salt as coagulant, but the residual chromaticity is low (Fig.3). There are also differences in the coagulation effects when with or without coagulant aids. The coagulation effect of poly-aluminum chloride is better than that of aluminum sulfate. After adding polyacrylamide as coagulant aids, the alum floc will further increase, but if the amount of poly-aluminum chloride is too large, the color of the sewage will become yellow, not only the residual turbidity will not drop, but also the chroma will increase.

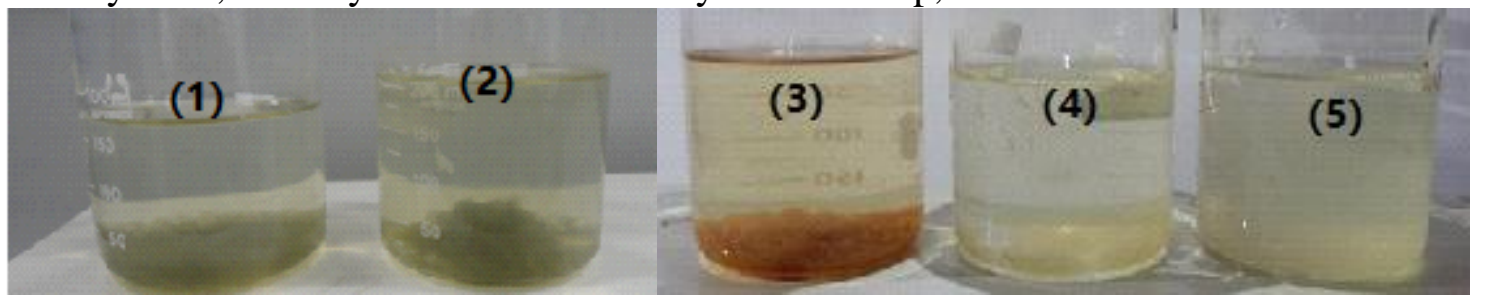

Fig.3 Coagulation effect of (1) ferrous salt, (2) ferrous salt with coagulant aids, (3) poly-aluminum chloride with polyacrylamide, (4) poly-aluminum chloride, (5) aluminum sulfate

Through comparison test, students have further perceptual knowledge about the water purification mechanism of coagulation and the main factors affecting the coagulation effect. In this process, students gradually enhance their awareness of active experiments and autonomous experiments, and establish their thinking habits of using engineering knowledge to solve engineering problems.

At the end of the experiment, students are required to compare their experimental results with the expected results; to write out the problems in the experiment, and solutions to this problems and related strategies, rethinking and questioning the various steps of the experiment; point out the deficiencies in the experiment and to improve the measures or suggestions, as well as their own harvest and experience.

When judging the experimental results, we should weaken "standard answers", highlight the experimental process and analysis, so that each group of students are focused on their own experiments and data analysis, avoiding the phenomenon of plagiarism report.

For another example, in the process of "removal of dissolved phosphorus from domestic sewage by adsorption and filtration process", some students found that after the adsorption of powdered activated carbon, the phosphorus content in the solution was not decreased, but increased; at the same time, the color of the solution was darkened. After analyzing the reason, it was found that powdered activated carbon was too fine to filtration clean, resulting in black activated carbon residue in the solution, interfering with the absorbance of the spectrophotometer, resulting in the determination of the phosphorus content in the solution increased; The modified experimental scheme was adsorbed by granular activated carbon, and then the phosphorus content in the solution was reduced. This improves the ability to analyze and solve problems by contrasting tests.

During the experiment, when encountered problems, students discussed actively, analyzed the causes of the problems, found solutions, and worked out a reasonable division of labor to overcome difficulties, and realized the perseverance and teamwork spirit of scientific research.

\section{Improve Scientific Literacy through Repeated Experimentation and Exploration}

The training of the whole process, such as consulting data, confirming scheme and experiment operation, can not only arouse students' enthusiasm for scientific research, cultivate students' innovative consciousness, but also cultivate students' scientific working attitude and enhance their cooperative consciousness and team spirit. 
For example, in experiments "coagulation, adsorption treatment of coal washing wastewater", some students used spectrophotometry to test hexavalent chromium in raw water when the absorbance is negative, the analysis shows that the content of hexavalent chromium in coal washing wastewater is too low, exceeding the range of spectrophotometer. It is necessary to improve the experimental program to complete the experiment, either increase the concentration of coal in the waste water or prolong the time of coal washing, so that the hexavalent chromium in the coals can be fully dissolved into water. At the same time, guide students to think that the heavy metal in coal is less and difficult to dissolve into the water, so need to increase concentration or prolonged contact time to measure its content.

While doing the experiment "activated carbon adsorption treatment of COD in coal washing wastewater", another students found that the removal efficiency was not related to the dosage of activated carbon when the amount of powdered activated carbon was in the range of 100-500mg, the experimental results were difficult to explain, with which model can't draw a linear adsorption isotherm; At this time, the instructor guided the students to think that the adsorption capacity of powdered activated carbon is too strong, and the amount of activated carbon is too large, which results in the saturation of adsorption, so it is not easy to find the difference of COD adsorption between different dosages, thus we should reduce the dosage and adjust the experimental plan. When the dosage of powdered activated carbon was in the range of $20-80 \mathrm{mg}$, the dosage of potassium permanganate difference increases significantly, and the adsorption efficiency of COD increased with the increase of activated carbon dosage.

For another example, in the experiment "monitoring and control of eutrophication in the RIXIN lake water", some students were in accordance with the requirements of the activated carbon intermittent adsorption experiment in Water Pollution Control Engineering Experiment Book, respectively added powdered activated carbon such as $0 \mathrm{mg}, 100 \mathrm{mg}, 200 \mathrm{mg}, 300 \mathrm{mg}, 400 \mathrm{mg}$, then monitored the COD(C) of the sewage before and after adsorption. According to Freundlich experience formula, adsorption isotherms were plotted on double logarithmic coordinates.

The results of previous years experimental showed that the students could not get a beautiful straight line, the calculated slope and intercept each group is not same, there is no orderliness at all. After the reform of the research teaching, the instructor reminded the students to increase the experimental data points, from the original 4 point to the 5 or 6 point, use Langmuir and Freundlich models to process the data, so that the adsorption isotherm will be made a straight line. And the fitting results show that the Langmuir model is more suitable for experiment data (Fig.4).

Through such repeated experiments and exploration, students' interest in experiments will been improved, and scientific literacy will been cultivated, at the same time, research and learning ability will been tempered and improved.

Freundlich

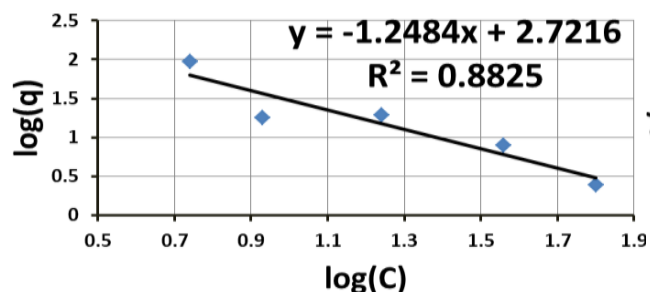

Fig.4 Adsorption isotherms obtained of Freundlich and Langmuir

\section{Langmuir}

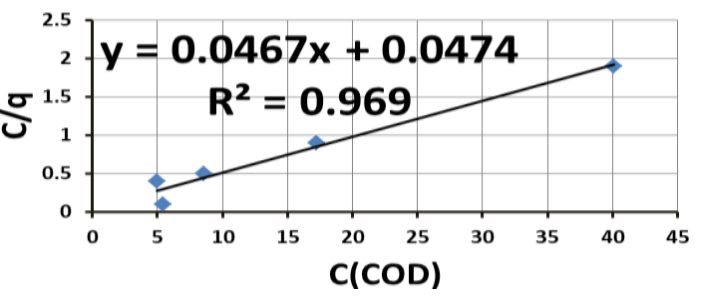


the students' harvests and experiences also have their own characteristics. Some students have the ability to understand the information of literature; Some students' experimental design and practical ability enhancement, and some students' level of experimental data processing, chart making, and paper writing have been improved. In generally, students' ability of logical reasoning, knowledge transfer and application, innovative thinking and solve environmental engineering practical problems have been trained and cultivated.

In grade 2013 and 2014, some students took part in state-level university student innovation projects, provincial and school level key innovation projects, provincial entrepreneurship competitions, school level energy conservation and emission reduction competitions, all achieved good results, while 5 patents were applied. It shows that the students' comprehensive ability has been exercised and improved.

\section{Acknowledgement}

This work was financially supported by North China Electric Power University Fund (No. 130017013) and Hebei Province Fund (No. 13001805).

\section{References}

[1] Anjun Chen. Discussion and practice based on project research teaching model, J. Heilongjiang Education. 11 (2011) 15-16.

[2] Peiyong Duan. Analysis of the research-based teaching model of cultivating innovative talents, J. Journal of Shandong University of Architecture. 04 (2012) 440-443+450.

[3] Jun Qiao, Fangyue He, Xianghong Huang. Research on the experimental teaching of the synthesis of ethyl acetate, J. Experimental Science and Technology. 06(2013) 65-66.

[4] Bang Zhang, Zhiqing Ni, Wei Wang, Xiaolei Wang. Teaching design and process management of comprehensive research experiment project, J. Experimental Technology and Management. 02 (2012) 159-161.

[5] Ping Mou, Jiexong Ding, Dan Qi, Lianzhong Guo, Li Du, Peifu Feng. Taking practical teaching as breakthrough point to improve students' researching ability, J. Experimental Technology and Management. 04 (2014) 157-159.

[6] Li Zhang, Qikun Zhu, Yanfan Bi, Xunming Xu. The application of PBL in the practice teaching of water pollution control engineering, J. Chemical Engineering Higher Education. 02 (2010) 90-94.

[7] Guqing Xiao, Yaoliang Wang, Dan Xie, Juanjuan Song. Discussion of environmental engineering experimental research-based teaching, J. China Electric Power Education. 01 (2013) 130-131.

[8] Chunhua Cao, Yazhen Wang, Li Liu. Practice and thinking of research-based teaching mode in "Material Chemistry" course, J. Guangzhou Chemical Industry. 44 (2016) 188-190.

[9] Yong Jin. Research-Type XRD optimization and practice of experimental teaching mode, J. Experimental Science and Technology. 14 (2016) 151-153+158. 